\title{
L'oxymore comme point de confluence des contraires : l'exemple de The Scarlet Letter de Nathaniel Hawthorne
}

\section{Jean Missud}

\section{(e) OpenEdition \\ Journals}

Édition électronique

URL : http://journals.openedition.org/esa/753

DOI : 10.4000/esa.753

ISSN : 2650-2623

Éditeur

Société de stylistique anglaise

Édition imprimée

Date de publication : 1 décembre 2016

Pagination : 105-119

ISBN : 978-2-36442-075-5

ISSN : 2116-1747

Référence électronique

Jean Missud, "L'oxymore comme point de confluence des contraires : I'exemple de The Scarlet Letter de Nathaniel Hawthorne ", Études de stylistique anglaise [En ligne], 10 | 2016, mis en ligne le 19 février 2019, consulté le 19 avril 2019. URL : http://journals.openedition.org/esa/753 ; DOI : 10.4000/esa.753 


\section{L'oxymore comme point de confluence des contraires : 1'exemple de The Scarlet Letter de Nathaniel Hawthorne}

Jean Missud

Aix Marseille Univ, LERMA, Aix-en-Provence, France

\section{Introduction}

L'oxymore est par définition une figure de l'impossible, de l'ineffable. Là où la langue ne peut être qu'approximative se trouve l'oxymore qui tend à exprimer l'indicible. Il n'est alors que peu étonnant de retrouver cette figure de style dans The Scarlet Letter qui est un roman dans lequel la parole se retrouve prisonnière. Hawthorne est un auteur qui, dans d'autres écrits tels que The Minister's Black Veil ou Young Goodman Brown, fait appel à des situations dans lesquelles il est impossible pour les personnages de s'exprimer, ces derniers étant forcés d'avoir recours aux masques, aux faux-semblants et aux non-dits. Plus encore que les silences, c'est la dualité des voix qui marque les personnages des œuvres de Hawthorne qui attire l'attention : que ce soit pour masquer une véritable identité ou pour échapper à sa destinée, un personnage hawthornien aura souvent comme outil d'expression le double-sens et l'association des contraires pour mettre ses rivaux sur une fausse piste ou pour écarter les soupçons qu'on lui porte. Ces thèmes sont récurrents chez Hawthorne. C'est ce que dit Sylvie Mathé : "What has been variously qualified as 'the device of multiple choice', 'the formula of alternative possibilities', or 'addiction to multiple interpretations' is undoubtedly Hawthorne's trademark " (1992, 610).

Dans le Boston puritain du XVII ${ }^{\mathrm{e}}$ siècle, The Scarlet Letter décrit une femme qui a donné naissance à une enfant après avoir eu une aventure avec un homme vivant aussi dans la colonie, alors que son mari 
est censé être perdu en mer. Mais ce mari apparaît dès les premières pages du roman: on voit donc un triangle amoureux se dessiner, dans un contexte historique et social qui fait de la naissance de cette enfant hors des liens du mariage un péché impardonnable. Punie par la communauté, Hester est désormais exclue de la vie de la colonie, murée dans un silence forcé et devra porter une lettre écarlate sur son habit, la lettre A pour ADULTERESS.

Dès lors, que ce soit pour masquer une véritable identité ou pour échapper à sa destinée, un personnage hawthornien aura souvent comme outil d'expression le double-sens et le mariage des contraires pour rester silencieux face aux questionnements d'autrui : ces stratégies vont trouver avec l'oxymore un lieu où la parole silencieuse s'exprimera à plein. La figure de l'oxymore et les exemples de cette figure présents dans The Scarlet Letter vont fonctionner comme un point d'ancrage des sentiments contraires qui animent les personnages tour à tour. Les dilemmes et les mensonges au cœur de l'intrigue de ce roman vont venir s'ancrer dans la figure oxymorique, parfait outil de l'expression de ce qui ne se dit pas dans une colonie puritaine américaine du XVII ${ }^{\mathrm{e}}$ siècle. Tel le trait d'union qui marie deux éléments de la langue, l'oxymore va venir unir les antonymes, à l'image de Hawthorne qui unit la femme pécheresse et la figure religieuse de la colonie.

Comment les structures oxymoriques, quelle que soit la forme syntaxique adoptée, permettent-elles à Hawthorne dans ce roman d'exprimer à la fois l'indicible et les dualités qui déchirent des personnages victimes de situations d'où il leur est impossible de s'extirper? La première étape consistera à tenter de circonscrire la forme oxymorique, puis nous verrons comment ses caractéristiques s'appliquent dans le roman.

\section{Qu'est-ce qu'un oxymore?}

L'oxymore est un élément de la stylistique peu étudié, comme le souligne notamment Michèle Monte :

Le terme même d'oxymore, devenu courant, n'a fait son apparition dans les traités de rhétorique que relativement récemment. Il existe pour désigner le même procédé un autre terme, celui d'alliance de mots (à comprendre comme une alliance de mots contradictoires) qui a la faveur de Duprez (1980) et qui seul figure dans le Larousse du $\mathrm{XX}^{\mathrm{e}}$ siècle en 1932. $(2008,37)$ 
Ces éléments expliquent peut-être en partie pourquoi trouver deux définitions identiques de cette forme est difficile; il semble qu'il faille se rattacher au contexte d'apparition de la forme pour justifier ou non de la présence d'un oxymore dans le texte. Le fait que les frontières soient minces avec le paradoxe, notamment, fait que la part d'interprétation du lecteur ne sera pas un facteur négligeable.

Michèle Monte, Patrick Hughes et Gilles Mathis sont ceux qui ont le mieux cerné la forme en l'abordant sous plusieurs aspects. Pour Monte, l'oxymore se définit ainsi : "L'oxymore [...], par le rapprochement au sein d'une même structure syntaxique de deux termes de sens opposé, propose une définition paradoxale d'un même objet de discours" (2008, 37). Toujours à propos d'une définition du terme oxymore, Patrick Hughes fait référence à son étymologie :

Oxymoron is itself an oxymoron. In Ancient Greek oxus means sharp and moros means dull. Thus an oxymoron is a sharp dullness. The terms are to be taken metaphorically: sharp as a metaphor for clever or wise, and dull as a metaphor for stupid or foolish. So oxymoron means foolish wise or silly clever. $(1984,125)$

L'on voit que l'oxymore implique la coexistence, dans un même syntagme ou un même énoncé, de deux points de vue apparemment contradictoires qui convergent à propos d'un même objet du monde ou d'un même événement. Mathis dit de l'oxymore : "[Il s'agit] d'une figure qui suppose la coexistence de deux contradictoires dans un même objet [...], le résultat d'une illusion d'optique ou de toute autre illusion sensorielle, ou le fruit de l'imagination " $(1990,1)$. Affirmation et négation sont donc mises côte à côte au sein de l'oxymore. L'auteur insiste sur le fait que l'oxymore est là pour tenter de combler un vide dans la langue :

L'oxymore témoigne d'une carence de la langue et nous parle d'une absence en même temps que d'une présence, celle d'un mystère [...]. C'est la figure de l'indicible, de l'impossible, de l'extrême du langage [...]. L'oxymore est une structure linguistique fondée sur la conjonction des contradictoires. (Mathis 1990, 1)

L'oxymore tente de nommer ce qu'il est impossible de nommer, représentant "l'écart maximum du point de vue sémantique et en même temps l'écart minimum du point de vue syntaxique par son raccourci fulgurant. Il est en somme un grand petit écart " (Mathis 1990, 1). 
L'esprit de contradiction présent dans la nature même de l'oxymore se retrouve dans ce que Mathis appelle habilement un "grand petit écart ", c'est-à-dire que l'écart de sens qui sépare les termes qui sont associés est contradictoire par rapport à la distance syntaxique qui sépare ces deux termes qu'a priori tout oppose. La démonstration de Mathis prouve que la dualité qui existe dans le langage de l'homme est pleine de contradictions tant ce dernier éprouve la nécessité de dire, de nommer, quitte à faire appel à des outils au sein desquels les paradoxes et les contradictions dominent.

Mathis souligne par ailleurs que l'oxymore s'oppose à l'antithèse et au paradoxe :

La définition logique de l'oxymore oppose en général antithèse, paradoxe et oxymore et recourt volontiers aux symboles aristotéliciens $\mathrm{A}$ et non-A :

Antithèse : A n'est pas non-A.

Paradoxe : A n'est pas A.

Oxymore : A est non-A. (Mathis 1988, 5)

Il tente une esquisse de typologie grammaticale de l'oxymore dans la langue anglaise, qui se présente comme suit :

- Adjectif + nom : loving hate, sweet unrest

- Nom + nom : life in death $(\mathrm{N}$ prep $\mathrm{N})$, conqueror and captive $(\mathrm{N}$ and $\mathrm{N})$

- Adjectif + adjectif : bitter-sweet (Adj-Adj), sweet and sad (Adj and Adj)

- Adverbe + adjectif : falsely true, unkindly kind

- Verbe + verbe : I burn and freeze

- Verbe + adverbe : supinely reign

- Nom + adverbe : future now

- Nom + verbe : the Eternal, as he was dying... (Mathis 1990, 1)

Dans les structures [Nom+Nom] et [Adjectif+Adjectif], on remarque de légères différences de constructions dans les exemples pris par Mathis.

Avec la structure [Nom+verbe], on sent déjà un décollement de la forme prototypique ramassée de l'oxymore car on a une distance syntaxique plus marquée, plus développée, et la présence d'un verbe conjugué laisse à penser que l'expansion syntaxique dans l'oxymore est acceptée par Mathis.

La structure [Adjectif + Nom] semble être celle qui répond le mieux à ce que l'on pourrait considérer comme étant une structure canonique de l'oxymore, c'est-à-dire l'oxymore dit ramassé, l'association simple de deux contraires où l'absence de toute autre sorte d'élément est à noter. Pour 
cette catégorie, Mathis crée une différence entre des «oxymores polarisés, primaires, mettant en jeu des extrêmes" comme loving hate et des " oxymores par dérivation métonymique" comme loving madness (1990, 21). On voit un continuum s'esquisser, qui irait de la simple mise côte à côte d'extrêmes à un type d'oxymore déjà plus subjectif puisque la folie pourrait être l'expression de l'amour. Il y a donc une différenciation qui est faite entre un oxymore composé de deux éléments opposés par leur sémantisme intrinsèque et un oxymore composé de deux éléments opposés par un sémantisme qui semble être davantage fait de subjectivité. On pourrait également parler d'un continuum qui irait de l'oxymore le plus ramassé à des oxymores plus dilués lorsqu'ils ont recours à la coordination - sweet and sad - au trait d'union - bitter-sweet - ou même à des structures verbales conjuguées - the Eternal, as he was dying. D'où l'importance capitale du contexte, comme nous le verrons dans notre étude de The Scarlet Letter, où certains oxymores apparaîtront comme dilués, et parfois affaiblis, par un étirement syntaxique.

\section{La présence oxymorique dans The Scarlet Letter}

\section{Hester Prynne}

Hester Prynne, personnage sur lequel l'attention est le plus souvent portée dans The Scarlet Letter, est la "cible " d'un très grand nombre des oxymores utilisés par l'écrivain.

Le premier exemple d'oxymore que nous proposons d'étudier est le suivant :

Those who had before known her, and had expected to behold her dimmed and obscured by a disastrous cloud, were astonished, and even startled, to perceive how her beauty shone out and made a halo of the misfortune and ignominy in which she was enveloped. It may be true that, to a sensitive observer, there was something exquisitely painful in it. (The Scarlet Letter $\left.{ }^{1}, 40\right)$

Nous avons un oxymore qui réunit l'adverbe positif exquisitely (de exquisite : exquis, raffiné, intense) et l'adjectif négatif, painful. Dans cette première occurrence, est présent un élément qui se révélera être capital par

1 Noté désormais TSL. Les éléments surlignés dans les citations sont de notre fait. 
L'OXYMORE COMME POINT DE CONFLUENCE DES CONTRAIRES :

L'EXEMPLE DE THE SCARLET LETTER DE NATHANIEL HAWTHORNE

la suite : la contradiction que porte en elle Hester Prynne, accusée et publiquement humiliée, mais d'une beauté incroyable. Hester est montrée du doigt et est traitée comme une pestiférée, mais, contrairement à ce qu'ils s'attendaient à ressentir, les membres de la communauté réunis pour l'humilier sont abasourdis par la beauté naturelle que dégage ce personnage pourtant coupable. La réunion du dégoût créé par l'acte dont elle est accusée et de la fascination créée par son aura se retrouve à merveille dans l'association exquisitely painful.

Plus tard, lorsqu'Hester quitte la prison, faisant face au monde extérieur, nous lisons : "Then, she was supported by an unnatural tension of the nerves, and by all the combative energy of her character, which enabled her to convert the scene into a kind of lurid triumph " (TSL, 55). L'oxymore consiste en la réunion de l'adjectif lurid et du substantif triumph, deux termes opposés et unis en un seul groupe nominal. Notons la présence de a kind of en début d'oxymore qui le dilue afin de renforcer l'idée de trouble, comme si le narrateur ne trouvait pas les mots pour décrire de manière exacte ce qu'il voit. À l'image de l'exemple précédent où le qualifié était something, a kind of indique que pour l'instant les deux oxymores servent à qualifier le vague, l'imprécis. Hawthorne utilise ici les oxymores à propos d'Hester Prynne comme des figures de l'approximativement construit. Cela peut être dû soit au statut social difficilement définissable d'Hester dans la colonie, soit à la préface du roman qui indique la manière dont le narrateur se procure les informations à propos des scènes qu'il relate : il trouve des éléments du XVII ${ }^{e}$ siècle relatant l'histoire de la 'Lettre Écarlate' se basant sur des récits oraux transmis de génération en génération. Hawthorne trouve peut-être là le moyen de nous dire qu'il ne prend pas totalement à son compte la véracité de ce qu'il décrit deux siècles après les faits. Comme le souligne Sylvie Mathé, Hawthorne est un auteur qui, dans The Scarlet Letter notamment, se désengage de ce qu'il avance et ne prend jamais véritablement parti. L'oxymore serait alors vu comme une figure du dédouanement, de l'éloignement de la vérité (1992, 610).

Hester s'interroge sur le pouvoir qu'a la lettre écarlate sur sa personnalité et sur la manière dont la communauté la perçoit - ou plus précisément, sur le fait que la punition qu'elle porte lui permet de voir les autres différemment. Un extrait du roman illustre ceci :

Walking to and fro, with those lonely footsteps, in the little world with which she was outwardly connected it now and then appeared to Hester-if altogether fancy, it was nevertheless too potent to be resisted-she felt or 
fancied, then, that the scarlet letter had endowed her with a new sense. She shuddered to believe, yet could not help believing, that it gave her a sympathetic knowledge of the hidden sin in other hearts. She was terrorstricken by the revelations that were thus made. What were they? Could they be other than the insidious whispers of the bad angel, who would fain have persuaded the struggling woman, as yet only half his victim, that the outward guise of purity was but a lie, and that, if truth were everywhere to be shown, a scarlet letter would blaze forth on many a bosom besides Hester Prynne's? Or, must she receive those intimations-so obscure, yet so distinct - as truth? In all her miserable experience, there was nothing else so awful and so loathsome as this sense. It perplexed, as well as shocked her, by the irreverent inopportuneness of the occasions that brought it into vivid action. Sometimes the red infamy upon her breast would give a sympathetic throb, as she passed near a venerable minister or magistrate. (TSL, 60)

L'opposition repose sur la juxtaposition de l'indétermination et de la netteté des messages reçus par Hester. L'oxymore oppose d'un côté la solitude que la punition reçue par Hester est censée provoquer dans la vie de la jeune femme, et d'un autre côté les connexions involontaires quasisurnaturelles que lui apporte la lettre écarlate. On a un exemple d'oxymore typiquement hawthornien, dilué (certains diront affaibli) par so et yet so. Le style du paragraphe tout entier correspond au style d'Hawthorne : sitôt un élément annoncé, le voici révoqué et amendé. Il peine à peindre des personnages aux sensations définitives, et l'oxymore - ainsi que les nombreuses virgules, tirets, connecteurs contrastifs et négatifs fonctionne à merveille ici pour décrire les objets du discours par ce qu'ils sont autant que par ce qu'ils ne sont pas, pour souligner l'absence autant que la présence. L'oxymore est inscrit dans un paragraphe que l'on pourra considérer comme une épanorthose, cette "figure par laquelle le locuteur ou le scripteur corrige, amende, précise, nuance et modifie sans cesse ce qu'il dit ou vient de dire ou d'affirmer précédemment ", selon Bruno Monfort (2005, 78-79). On note le nombre important de revirements de points de vue en un seul paragraphe : if, nevertheless, or, yet, as well as, phrases interrogatives, tirets...

L'écrivain donne à "voir " plusieurs aspects d'Hester, tant et si bien que le lecteur ne peut pas parvenir à détecter le vrai du faux, le ressenti du surnaturel, tant sa "vue» est brouillée par la voix du narrateur, et l'insertion d'oxymores permet de dire ce qui ne se dit pas en laissant le flou planer au-dessus de cette femme coupable. 
L'OXYMORE COMME POINT DE CONFLUENCE DES CONTRAIRES :

L'EXEMPLE DE THE SCARLET LETTER DE NATHANIEL HAWTHORNE

\section{Pearl}

En ce qui concerne l'enfant diaboliquement angélique, Pearl, une enfant qui peut être considérée elle-même comme figure oxymorique tant elle unit d'une part l'image du péché et d'autre part l'image de la pureté de l'enfant. Sylvie Mathé le note ainsi :

Pearl, as a living embodiment of the Scarlet Letter, is herself an oxymoron (...): she unites the outward saint Dimmesdale, with the outward sinner Hester, in what is precisely an impossible bond in the eyes of the Puritan society $(1992,622)$.

Les sentiments qui animent la mère vis-à-vis de sa fille sont contradictoires, Hawthorne trouvant un oxymore pour évoquer cela :

Her only real comfort was when the child lay in the placidity of sleep. Then she was sure of her, and of her tasted hours of quiet, sad, delicious happiness; until- perhaps with that perverse expression glimmering from beneath her opening lids-little Pearl awoke! (TSL, 63-64)

Le bonheur qui habite Hester est décrit à l'aide d'un oxymore, une figure qui unit l'adjectif sad et le nom happiness. Cela traduit la position d'Hester vis-à-vis de sa fille, qu'elle aime mais qu'elle craint. Notons la complexité de la caractérisation des personnages, réalisée par le biais un trio d'adjectifs où l'oxymore pénètre la structure de base. Hawthorne vient insérer un oxymore qu'il espère voir passer inaperçu par l'insertion d'un qualificatif négatif contradictoire au cœur d'une série d'adjectifs positifs.

La relation ambiguë qui lie Pearl et sa mère est mise en avant dans un autre oxymore : "She is my happiness! She is my torture, none the less! Pearl keeps me here in life! Pearl punishes me too! " (TSL, 76). Dans un moment où elle se retrouve sur la défensive, face à la possibilité de se voir enlever son enfant, Hester exprime de manière véhémente le fond de sa pensée, sans frein. C'est dans ces moments que s'exprime la vraie nature des sentiments, et ceux qu'exprime Hester sont contradictoires et difficilement déterminables. L'enfant est à la fois un bonheur et une torture, un moyen de rester en vie mais également une punition. Le premier oxymore consiste en la qualification de $S$ he, à savoir Pearl, qui est affublée de deux noms contradictoires pour la qualifier : l'oxymore a pour sujet she et pour composantes happiness d'une part et torture d'autre part, l'objet she étant répété. L'objet Pearl dans le second oxymore est également répété, comme pour renforcer la dualité de l'énoncé : dans ce second cas, ce sont deux groupes verbaux qui viennent 
qualifier l'objet Pearl. L'oxymore (dilué syntaxiquement, proche du paradoxe) oppose la relation prédicative $<$ Pearl / keep me in life $>$ d'une part et la relation prédicative $<$ Pearl / punish me $>$ d'autre part. À propos de cet exemple, Mathé (qui considère cette occurrence comme un oxymore) fait cette observation : "The balanced exclamative structures of the beginning, though weakened by the concessive use of the adverbs none the less and too can be read as a subdued form of oxymora pointing to the bond linking mother and child" (Mathé 1992, 622).

En ce qui concerne la forme de cet énoncé, nous nous appuyons sur les conclusions de Mathis pour inclure cette occurrence dans notre étude :

[L'oxymore peut] s'inscrire éventuellement dans une suite binaire de phrases, car si l'oxymore rejette pour lui-même les formes négative et interrogative, il admet en revanche l'exclamative en deux indépendantes, juxtaposées ou coordonnées, elliptiques ou non, qui ne sont à tout prendre que l'éclatement du syntagme de base $(1990,18)$.

\section{Chillingworth \& Dimmesdale}

Les descriptions qui sont faites des deux personnages masculins, Dimmesdale et Chillingworth, n'échappent pas aux oxymores d'Hawthorne pour traduire l'ambivalence de leur personnalité respective.

Dans un moment clé du roman, le moment de la découverte sur la poitrine du pasteur de la lettre $A$, jumelle de celle brodée sur le vêtement d'Hester, le mari a une première réaction naturelle, sans barrière ni arrière-pensée, qui est révélatrice de son caractère ambivalent au moment où il découvre l'impensable vérité qu'il redoutait : "But with what a wild look of wonder, joy, and horror! With what a ghastly rapture [he] stamped his foot upon the floor! " (TSL, 92). Dans cet énoncé, le médecin est animé d'une vision à la fois d'émerveillement, de joie et d'horreur qui le pousse dans un état d'hystérie jamais vu auparavant chez le discret scientifique. Comme le note Sylvie Mathé: "Chillingworth [...] is an incarnation of the oxymoric principle of reversibility, torturer and victim, destroyer and destroyed, sadist and masochist " (1992, 624).

Plus loin, Hester accuse Chillingworth d'être le responsable des souffrances de celui qui est le père de son enfant. À cette occasion, pour décrire la relation difficilement déterminable qui lie les deux hommes, l'auteur a recours à l'oxymore :

You tread behind his every footstep. You are beside him, sleeping and waking. You search his thoughts. You burrow and rankle in his heart! Your 
L'OXYMORE COMME POINT DE CONFLUENCE DES CONTRAIRES :

L'EXEMPLE DE THE SCARLET LETTER DE NATHANIEL HAWTHORNE

clutch is on his life, and you cause him to die daily a living death, and still he knows you not. (TSL, 111)

Cet oxymore est double : dans un premier temps, l'oxymore réunit le verbe die et l'adverbe daily puis dans un deuxième temps, il unit living et death. L'idée sous-jacente est de démontrer que la vie qu'inflige Chillingworth à Dimmesdale est un non-sens tant les souffrances causées sont grandes. La répétition du son [d] n'est pas non plus anodine et associe phonologiquement daily au couple die et death.

Plus tard, il est question des souffrances de Dimmesdale, dont Hawthorne parle en ces termes : "Unless it avails him somewhat that he was broken down by long and exquisite suffering; that his mind was darkened and confused by the very remorse which harrowed it " (TSL, 129). L'oxymore met en jeu l'opposition entre les deux adjectifs qualificatifs qui portent sur le nom suffering, d'une part l'adjectif long et d'autre part l'adjectif exquisite. Hawthorne a de nouveau recours à exquisite et semble vouloir unir à distance le exquisitely painful de Hester et l'exquisite suffering de celui qu'elle aime. L'utilisation de la conjonction de coordination and n'était ici pas obligatoire, le recours à la juxtaposition aurait aussi bien décrit la situation au lecteur. On peut postuler l'idée selon laquelle and est utilisé pour deux raisons : d'abord pour rendre visible le lien qui unit les contraires, un lien qu'une juxtaposition plus classique aurait effacé ; puis pour dresser un parallèle avec le couple darkened and confused qui apparaît ensuite et qui est lui logiquement articulé. Donc non seulement l'oxymore oppose long et exquisite, mais l'oxymore entier est opposé au couple darkened/confused, comme pour souligner les souffrances ambivalentes éprouvées par Dimmesdale alors que son esprit est sans aucun doute obscurci et confus. C'est sur les conséquences des souffrances que Hawthorne insiste, plutôt que sur les raisons de ces dernières. Les souffrances psychiques et physiques dont souffre le pasteur sont longues mais exquises : telle est la force de la contradiction qui anime le pasteur, pris entre sa fonction religieuse au sein de la communauté et sa volonté de parler. Ici, Hawthorne dresse le portrait d'un homme tiraillé entre deux extrêmes, faisant face à un choix impossible à faire entre deux solutions qui n'en sont pas.

La voix de Dimmesdale est un élément-clé dans The Scarlet Letter. En effet, le pasteur est celui dont les souffrances physiques sont les plus évidentes, des souffrances que l'on pourrait qualifier de psychosomatiques tant elles sont le résultat de la culpabilité qui ne peut s'exprimer sous 
forme de mots et qui se manifeste par des souffrances du corps. Les maux dont est victime Dimmesdale ont une influence directe sur les mots que prononce le pasteur. L'exemple le plus parlant est le Sermon de l'Élection durant lequel les mots sont qualifiés d'inaudibles. Le Sermon de l'Élection est à n'en pas douter le paroxysme du roman, il n'est alors que peu surprenant de retrouver des oxymores dans un discours qui n'en est pas un, dans un moment de révélation qui n'en sera pas un. Dans le roman, la voix du pasteur joue un rôle prépondérant tant les mots prononcés, pourtant impossibles à distinguer, tombent dans une oreille déjà conquise. Les mots de Dimmesdale qui sont censés être l'outil de la révélation de la vérité, ne remplissent pas le rôle attendu, et c'est par un oxymore qu'Hawthorne l'exprime :

Hester Prynne listened with such intenseness, and sympathized so intimately, that the sermon had throughout a meaning for her, entirely apart from its indistinguishable words. These, perhaps, if more distinctly heard, might have been only a grosser medium, and have clogged the spiritual sense (TSL, 154).

Il y a des exemples d'oxymore qui ne sont oxymores que dans le contexte de The Scarlet Letter. Autrement dit, le contexte dans lequel la figure de style s'inscrit fait de l'élément étudié un oxymore. L'auteur nous dit que si les mots du pasteur dans son sermon étaient audibles, ils perdraient leur sens spirituel. Pour Hester, tout le sermon a une signification, le discours lui parle en quelque sorte, mais pas les mots qui sont impossibles à identifier. L'oxymore souligne l'impossible existence de mots qui ne peuvent pas être entendus ou distingués par l'auditoire, ainsi que le caractère indescriptible de la relation qui unit le pasteur et l'Adultère, une relation qui ne doit pas être mise en mots et qui est donc codée pour ne parler qu'à un membre de la congrégation, Hester. Dans un moment de souffrance tant physique que psychologique - "While thus suffering under bodily disease, and gnawed and tortured by some black trouble of the soul" (TSL 93) - les mots de Dimmesdale ont perdu de leur clarté ; ce qui étonne, c'est que les mots, bien qu'inaudibles, ne peinent pas à convaincre.

Ce passage est caractéristique des techniques utilisées par Hawthorne dans le roman : il place volontairement Hester loin de l'endroit d'où s'exprime son amant, mais elle parvient tout de même à être transportée par le flot de paroles émanant du pasteur. Ou, plus précisément, elle réussit à percevoir le non-dit dans les paroles qui arrivent 
L'OXYMORE COMME POINT DE CONFLUENCE DES CONTRAIRES :

L'EXEMPLE DE THE SCARLET LETTER DE NATHANIEL HAWTHORNE

jusqu'à elle, elle parvient à détecter la douleur dans ces mots nonprononcés ; d'une manière révélatrice, cette voix qui conquiert les foules peine à convaincre un lecteur qui est dans la même position physique qu'Hester, c'est-à-dire loin du pasteur. Ce ne sont donc pas les mots qui touchent Hester mais les émotions contenues dans l'éloquence d'une voix au message à peine audible ; les manques et les blancs jouent un rôle-clé dans un roman où indétermination et faux-semblant dominent. Il est intéressant de noter que dès le chapitre 3, lors d'une des premières prises de parole du pasteur, Hawthorne soulignait déjà le fait que : "The feeling that it [the pastor's voice] so evidently manifested, rather than the direct purport of the words, caused it to vibrate within all hearts, and brought the listeners into one accord of sympathy " (TSL, 49). Pour Michèle Bonnet :

C'est [...] à l'occasion des sermons de Dimmesdale, là où Hawthorne insiste sur le pouvoir de la voix pure, qu'il exprime de la façon la plus explicite sa défiance à l'égard du langage verbal. [...] Les mots, ajoute le narrateur, ne sont qu'un "instrument grossier». [...] Les paroles du ministre sont "indistinctes" derrière sa voix [...] et sans effet sur ses auditeurs. Si ceux-ci sont profondément ébranlés, ce n'est pas, à coup sûr, par ses paroles, car ils "n'ont rien saisi du langage dans lequel parlait le pasteur ». [...] Ce n'est donc pas dans les mots, matériau inerte du discours, qu'il faut chercher la source de l'éloquence du pasteur. (2005, 66-67)

En toute fin de roman, le pasteur prend la place symbolique d'Hester sur l'échafaud, et nous relevons : "By bringing me hither, to die this death of triumphant ignominy before the people! " (TSL, 162). L'oxymore consiste en la caractérisation du nom ignominy par l'adjectif qualificatif triumphant. Comme le souligne Mathé, "Dimmesdale's oxymoric destiny culminates at the scaffold "(1992, 621). L'aspect double de la vie menée par Dimmesdale se retrouve dans sa mort. Notons que Dimmesdale et Hester sont enfin liés à la conclusion de ce roman, non pas par la relation qu'ils ne pouvaient pas vivre, mais par les mots qu'ils utilisent dans les situations désespérées auxquelles ils sont confrontés. Si Dimmesdale parle de "triumphant ignominy " (TSL, 162), Hester faisait quant à elle référence à un "lurid triumph" (TSL, 55) accompagné d'une "ignominy in which she was enveloped" (TSL, 40). Dimmesdale et Hester sont les victimes d'oxymores portant sur les mêmes termes, qui se font écho et se répondent d'une extrémité à une autre du texte. 


\section{Conclusion}

The Scarlet Letter est un roman qui met en scène des personnages qui sont tous, à un moment ou à un autre de leur évolution au sein du roman, confrontés à des tiraillements, des prises de décision impossibles, des choix douloureux, et surtout font face à l'impossibilité de parler, l'impossibilité de révéler l'intégralité des sentiments qui les animent. Cela se traduit de plusieurs manières, d'abord dans leurs comportements respectifs, faits de secrets, mensonges, revirements de situation, changements d'avis. D'un point de vue littéraire, cela se traduit par des rencontres secrètes, des conversations privées, des discours intérieurs. D'un point de vue stylistique, cela se répercute par l'utilisation de formes linguistiques et grammaticales traduisant l'impossibilité de parler, ou plutôt l'impossibilité de dire la vérité telle qu'elle est ressentie. Pour communiquer au lecteur l'impossibilité de communiquer, Nathaniel Hawthorne a recours à plusieurs procédés grammaticaux et stylistiques, notamment l'oxymore. Cette figure de style associe des éléments contraires d'une manière extrêmement rapprochée. Elle confronte, par l'absence d'éloignement qu'elle propose, une «jumellisation " des contraires, une juxtaposition d'éléments a priori impossibles à juxtaposer. Nous avons vu à quel point le rapprochement syntaxique des contraires dans la forme stylistique qu'est l'oxymore rappelle le rapprochement physique des contraires dans l'esprit des personnages du roman.

La présente étude a permis de prouver que l'utilisation des oxymores par Hawthorne dans The Scarlet Letter était très fréquente ; pourquoi cette figure de style est-elle si souvent utilisée dans ce roman? Quelles en sont les conséquences sur le lecteur?

Une première hypothèse serait que l'auteur offre une multitude de possibilités, comme s'il proposait différents scénarios pour chaque objet de son récit, et qu'il s'agirait alors pour chaque lecteur de choisir entre un aspect ou un autre de chaque personnage faisant l'objet d'un ou plusieurs oxymore(s).

Une seconde hypothèse serait celle qui privilégierait l'idée selon laquelle il faudrait prendre chaque sujet comme un élément uni malgré sa diversité, l'oxymore étant la figure du discours qui permettrait de passer outre les frontières apposées par un langage qui peine à considérer la multiplicité des facettes présentes dans un objet. Il ne s'agirait donc pas d'additionner les éléments qui font cet objet, mais de le voir dans sa pluralité. 
Dans le texte, d'autres éléments trahissent la mise en mots de l'indicible, de l'indescriptible: Hawthorne lutte parfois pour trouver le moyen de qualifier ce qu'il voit. C'est notamment ce que trahit le recours à l'épanorthose. L'indétermination porte à certains moments non plus sur le sens à donner aux choses, mais sur l'existence même de celles-ci. Dès lors, l'oxymore, qui accolait deux contraires, ne paraît plus suffire à Hawthorne pour induire dans l'esprit du lecteur la remise en question de la réalité telle qu'elle semble se présenter de prime abord. C'est ce que confirme Bruno Monfort lorsqu'il écrit que :

Nombre d'épisodes du roman ne mettent pas en jeu une indécision épistémique [...] mais l'existence même de ce dont il est question, objet, acte ou événement. De ce point de vue, le roman serait beaucoup plus radical puisqu'il remettrait en question l'existence de ce qui est, ou plutôt semble être. $(2005,78)$

Si l'on étend la présente étude sur The Scarlet Letter aux préfaces et essais de son auteur, la notion de voile et de double-langage - au travers de l'oxymore, de l'épanorthose et de diverses techniques littéraires (mises en scène, malentendus, rencontres secrètes...) - domine l'écriture du natif de Salem. C'est ce que Thomas R. Moore indique dans sa préface :

The recognition of language as a shifting entity - sometimes obscuring, sometimes illuminating the truth - is a thread weaving itself through the long fabric of Hawthorne's work. Although he is known as a writer of fiction, with veils and ambiguities shrouding his discourse in the tales and romances, it is the sketches, prefaces and essays that fully reveal his preoccupation with the slippery, untrustworthy nature of language and rhetoric. (Moore 1994, xi)

Ce passage nous pousse à vouloir élargir le terrain d'étude de l'écriture de Hawthorne, qui ne révèle pas tous ses secrets dans The Scarlet Letter. 


\section{BIBLIOGRAPHIE}

\section{CORPUS}

HAWTHORNE, Nathaniel. 2005 [1850]. The Scarlet Letter and Other Writings. New York : Norton Critical Edition.

\section{RÉFÉRENCES}

BONNET, Michèle. 2005. "La voix éloquente, ou comment dire ce qui ne peut se dire ». Sillages Critiques $7: 63-86$.

HUGHES, Patrick. 1984. More on Oxymoron. London: Jonathan Cape.

MATHÉ, Sylvie. 1992. "The Reader May Not Choose: Oxymoron as Central Figure in Hawthorne's Strategy of Immunity from Choice in The Scarlet Letter». Style 26 : 605-633.

MATHIS, Gilles. 1988. "L'oxymore, essai d'analyse». Bulletin de la Société de Stylistique Anglaise 10 : 27-55.

MATHIS, Gilles. 1990. "Oxymore et expansion ». Bulletin de la Société de Stylistique Anglaise 12 : 33-68.

MONFORT, Bruno. 2005. "Le style de Hawthorne : de l'oxymore à l'épanorthose ». The Scarlet Letter : 61-80.

MONTE, Michèle. 2008. "Le jeu des points de vue dans l'oxymore : polémique ou reformulation?». Langue Française 160 : 37-53.

MOORE, Thomas R. 1994. A Thick and Darksome Veil: The Rhetoric of Hawhorne's Sketches, Prefaces and Essays. Boston: Northeastern University Press. 